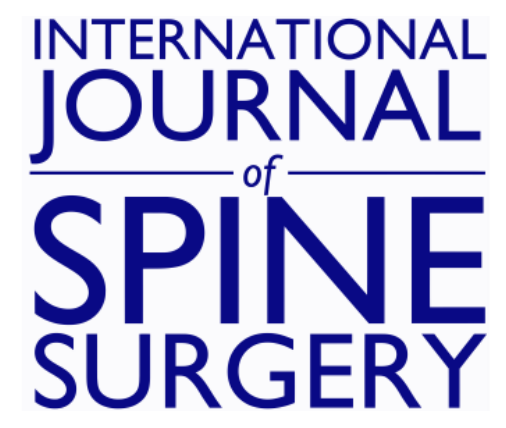

\title{
Fortifying the Bone-Implant Interface Part 2: An In Vivo Evaluation of 3D-Printed and TPS-Coated Triangular Implants
}

Regina F. MacBarb, Derek P. Lindsey, Shane A. Woods, Peggy A. Lalor, Mukund I. Gundanna and Scott A. Yerby

Int J Spine Surg 2017, 11 (3)

doi: https://doi.org/10.14444/4016

http://ijssurgery.com/content/11/3/16

This information is current as of April 26, 2023.

Email Alerts Receive free email-alerts when new articles cite this article. Sign up at:

http://ijssurgery.com/alerts

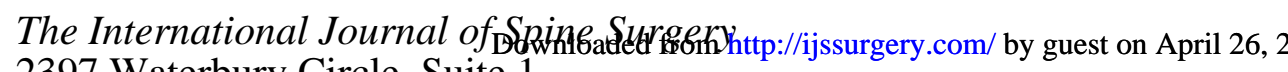
2397 Waterbury Circle, Suite 1,

Aurora, IL 60504, Phone: +1-630-375-1432

(C) 2017 ISASS. All Rights Reserved. 


\section{Fortifying the Bone-Implant Interface Part 2: An In Vivo Evaluation of 3D-Printed and TPS-Coated Triangular Implants}

Regina F. MacBarb, PhD, ${ }^{1}$ Derek P. Lindsey, MS, ${ }^{1}$ Shane A. Woods, MS, ${ }^{2}$ Peggy A. Lalor, PhD, ${ }^{3}$ Mukund I. Gundanna, MD, ${ }^{4}$ Scott A. Yerby, PhD ${ }^{1}$ ${ }^{1}$ SI-BONE, Inc., San Jose, CA, USA, ${ }^{2}$ MPI Research, Mattawan, MI, USA, ${ }^{3}$ Histion LLC, Everett, WA, USA, ${ }^{4 B r a z o s ~ S p i n e, ~ C o l l e g e ~ S t a t i o n, ~ T X ~}$

\section{Abstract}

\section{Background}

Minimally invasive surgical fusion of the sacroiliac (SI) joint using machined solid triangular titanium plasma spray (TPS) coated implants has demonstrated positive clinical outcomes in SI joint pain patients. Additive manufactured (AM), i.e. 3D-printed, fenestrated triangular titanium implants with porous surfaces and bioactive agents, such as nanocrystalline hydroxyapatite (HA) or autograft, may further optimize bony fixation and subsequent biomechanical stability.

Methods

A bilateral ovine distal femoral defect model was used to evaluate the cancellous bone-implant interfaces of TPScoated and AM implants. Four implant groups ( $\mathrm{n}=6 /$ group/time-point) were included: 1 )TPS-coated, 2)AM, 3) $\mathrm{AM}+\mathrm{HA}$, and 4) $\mathrm{AM}+$ Autograft. The bone-implant interfaces of 6 - and 12 -week specimens were investigated via radiographic, biomechanical, and histomorphometric methods.

Results

Imaging showed peri-implant bone formation around all implants. Push-out testing demonstrated forces greater than $2500 \mathrm{~N}$, with no significant differences among groups. While TPS implants failed primarily at the boneimplant interface, AM groups failed within bone $\sim 2-3 \mathrm{~mm}$ away from implant surfaces. All implants exhibited bone ongrowth, with no significant differences among groups. AM implants had significantly more bone ingrowth into their porous surfaces than TPS-coated implants $(p<0.0001)$. Of the three AM groups, AM+Auto implants had the greatest bone ingrowth into the porous surface and through their core $(p<0.002)$.

\section{Conclusions}

Both TPS and AM implants exhibited substantial bone ongrowth and ingrowth, with additional bone through growth into the AM implants' core. Overall, AM implants experienced significantly more bone infiltration compared to TPS implants. While HA-coating did not further enhance results, the addition of autograft fostered greater osteointegration for AM implants.

\section{Clinical Relevance}

Additive manufactured implants with a porous surface provide a highly interconnected porous surface that has comparatively greater surface area for bony integration. Results suggest this may prove advantageous toward promoting enhanced biomechanical stability compared to TPS-coated implants for SI joint fusion procedures.

\section{Introduction}

Chronic low back pain (LBP) is a widespread public health burden with a tremendous socio-economic impact. Cross-sectional population-based surveys have demonstrated that $13.1 \%$ of U.S. adults age 20 -
69 suffer from LBP, with a higher prevalence in women. ${ }^{1}$ Health care costs for treatment and lost wages due to LBP are estimated to exceed $\$ 250$ billion annually in the U.S alone. ${ }^{2}$ The sacroiliac (SI) joint has been recognized as the source of pain in $15-$ $30 \%$ of LBP patients. ${ }^{3-7}$ When conservative approach- 
es fail, surgical intervention, such as a minimally invasive fusion procedure, may be a viable option.

Prospective clinical trials ${ }^{8-10}$ have shown high levels of pain relief and improvement in disability scores and quality of life in patients undergoing SI joint fusion with a series of titanium plasma spray (TPS) coated triangular implants placed transarticularly across the SI joint. Computed tomography scan follow-up in the two U.S. trials have shown high rates of bone adherence to implants and signs of bridging bone across the SI joint. ${ }^{8,10}$ A small number of subjects in the trials (2-3\%) experienced recurrent or persistent pain along with radiolucencies around the implants, particularly within the sacrum. In some cases, patients underwent revision surgery (typically placement of an additional implant, sometimes with removal of implants placed during the index procedure). The rate of revision surgery after SI joint fusion with triangular titanium implants is low $(<4 \%$ at 4 years, ${ }^{11}$ i.e., approximately $1 / 3$ of that after lumbar surgery $\left.{ }^{12}\right)$; however, there remains room for improvement. Methods to enhance the biological fixation between these SI joint fusion implants and the surrounding host bone presents as an opportunity to increase the long-term effectiveness of this procedure and to further reduce the rate of revision surgery.

Since the introduction of porous coatings into the orthopedic community in the late $1960 \mathrm{~s},{ }^{13}$ TPScoatings have seen the most widespread usage in hip and knee implants, and more recently in spine implants. ${ }^{14,15}$. Notable spinal applications include interbody fusion cages and artificial discs. ${ }^{16,17}$ TPS-coated surfaces are characterized by a graded distribution of pores ranging from $100-150 \mu \mathrm{m}$ at the surface that transition to a solid base ${ }^{18}$ typically resulting in highly variable surfaces with low pore interconnectivity that do not emulate native bone structure. ${ }^{14,19,20} \mathrm{Re}-$ cent articles on engineered bone scaffolds, however, suggest that highly interconnected porous surfaces with pore sizes ranging from $200-400 \mu \mathrm{m}$ and porosities of $45-65 \%$ are ideal for maximizing bone-implant integration. ${ }^{21,22}$ The recent advent of additive manufacturing, i.e. 3D-printing, allows for fabrication of implants with previously unachievable geometries that have more consistent and highly controlled porous surfaces. ${ }^{23}$ While additive manufactured
(AM) implants are now being used clinically, in situ data describing their osteointegration characteristics are limited.

Another means to elicit greater bone apposition to implant surfaces is to enhance surface bioactivity. Of the available methods, hydroxyapatite (HA) coatings have been the most extensively used for orthopedic implants. ${ }^{24}$ The most common method to HA-coat an implant is plasma-spraying. This line-of-site process, however, cannot uniformly penetrate a porous structure, and thereby results in variable surface coverage that often occludes desirable surface features. ${ }^{25}$ An alternative method is to deliver nanocrystalline HA particles via dip-coating precipitate. This method has been shown to foster early bone formation adjacent to smooth titanium and polyetheretherketone (PEEK) implants in vivo ${ }^{26,27}$ and to fully penetrate AM porous metal surfaces (see Part I). However, whether the addition of nanocrystalline HA to AM titanium porous implants promotes enhanced bone apposition in vivo remains unknown.

This study evaluated the bone-implant interface of TPS-coated implants and AM implants in an ovine femoral defect model. Control implants were TPScoated triangular implants FDA-cleared for SI joint fusion. The AM implants incorporated a printed porous surface designed to mimic cancellous bone architecture and an open fenestrated geometry that can accept bone graft. It was hypothesized that 1 ) the AM implants would display similar or enhanced biomechanical stability and bone integration compared to TPS-coated implants; 2) the AM implant design would allow for bone ongrowth and ingrowth to the porous surface and through the implant's core; and 3) the addition of nanocrystalline HA to the surface of the AM implants or placing autograft onto and into the AM implants would further promote osteointegration.

\section{Materials \& Methods}

\section{Implants}

Control implants were machined from wrought Ti6Al4V ELI and coated with a $0.75 \mathrm{~mm}$ thick commercially pure TPS-coating (iFuse Implant; SI- 
BONE, Inc. San Jose, CA). Test implants were additive manufactured via electron beam melting technology (Arcam AB, Mölndal, Sweden) using Ti6Al4V ELI powder per ASTM F3001. All implants were $7.0 \mathrm{~mm}$ in inscribed solid core diameter, triangular in cross-section, and $45 \mathrm{~mm}$ in length. AM implants were designed to have an open fenestrated structure and a $\sim 0.75 \mathrm{~mm}$ thick porous surface to offer greater available surface area for enhanced osteointegration (Figure 1). The porous surface was generated using parameters previously determined in vitro (see Part I) to have an average pore size of $300 \mu \mathrm{m}$ to promote osteointegration, ${ }^{22}$ and an average porosity of $60 \%$ to fall within the range of native cancellous bone ${ }^{28}$. AM implants were either investigated as is, coated with a nanocrystalline HA-coating $(\mathrm{AM}+\mathrm{HA})$, or augmented with autograft $(\mathrm{AM}+\mathrm{Au}-$ to). In total, there were 4 experimental conditions.

For AM+HA implants, a $\sim 20$ nm layer of HA was applied to implant surfaces using a dip-coating precipitate technique as previously described. ${ }^{26,27} \mathrm{AM}+$ Auto implants are described in the Animal Model section below. All implants were gamma-sterilized prior to use.

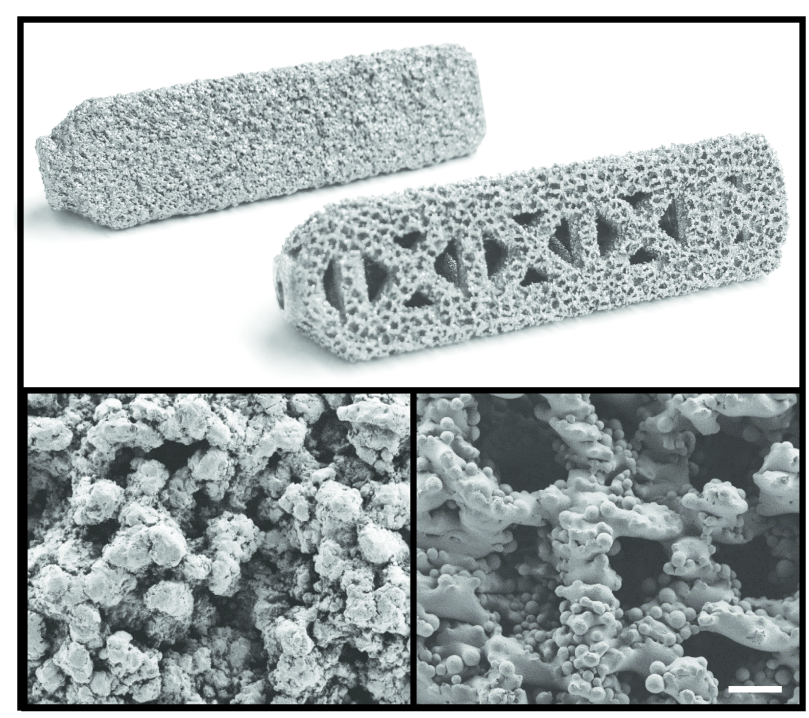

Fig. 1. Top: Images of the control TPS-coated implant (back) and additive manufactured (AM) implant (front). All implants were $45 \mathrm{~mm}$ long x 7.0 $\mathrm{mm}$ inscribed circular diameter. Control implants were comprised of a machined Ti6AI4V ELI core with a $0.75 \mathrm{~mm}$ thick commercially pure TPS-coating. AM implants were additive manufactured using Ti6Al4V ELI particles for both the base and porous surface structures. Bottom: Scanning electron microscopy images of the TPS (left) and AM (right) surfaces at $100 x$. Scale bar $=300 \mu \mathrm{m}$.

\section{Animal Model}

The study protocol was approved by the IACUC at MPI Research (Mattawan, MI). Implants ( $\mathrm{n}=6$ per group) were placed in 24 healthy skeletally mature female Suffolk sheep with each animal receiving implants from two different groups. Under general anesthesia, bilateral triangular distal femoral defects were created using a guide pin, trephine, and broach under fluoroscopic guidance such that the implants were predominantly placed in cancellous bone. For animals receiving an $\mathrm{AM}+$ Auto implant, bilateral bone cores obtained from the trephines were combined, ground, and pressed into the porous surface and open fenestrated windows. All implants were press-fit medial-to-lateral. Prophylactic pre- and post-operative antibiotics were administered. Once recovered from anesthesia, sheep were returned to their housings and were able to walk undisturbed on the first postoperative day. Sheep were maintained in housing with normal access to food and water until euthanasia at 6 or 12 weeks.

\section{Postmortem Evaluations}

At necropsy, radiographic images were obtained. Implants were removed $e n$-bloc and bisected (Figure 2A). Biomechanical push-out testing was performed using a uniaxial testing frame (Instron, series 566A) with a triangular ram and a base with a triangular clearance hole. A $10 \mathrm{~mm}$ thick section was obtained from the medial side of each specimen, where only cancellous bone surrounded the segment (Figure 2A). Each specimen was pushed out of cancellous bone at $5 \mathrm{~mm} / \mathrm{min}$ until fully separated, as previously described. ${ }^{29}$ Maximum push-out force, energy-tofailure, and ultimate shear strength were calculated (area for shear strength was calculated as the sum of the 3 nominal triangular faces). The lateral side of each implant was fixed in $10 \%$ neutral buffered formalin and submitted for microcomputed tomography $(\mu \mathrm{CT})$ and histopathology. For $\mu \mathrm{CT}$, tissue blocks were scanned at a resolution of $24.5 \mu \mathrm{m}^{3}$ (Scanco Micro-CT 100). Specimens were then embedded in methylmethacrylate. Sections were taken as close as possible to two targeted locations (A and B in Figure 2A) on all implants and stained with Stevenel's blue/ van Gieson stain. A blinded pathologist scored the presence of lamellar bone into quartiles to assess bone maturation at the surface of all implants and 
within the central region of the AM groups. Semiquantitative scoring was also conducted to assess necrosis and inflammation using a similar quartile scale. Quantitative histomorphometric analysis was performed on all slides to quantify 1 ) bone in contact with the periphery (i.e., bone ongrowth) of the implants, 2) bone area within the porous surfaces (i.e., bone ingrowth) of all implants and, and 3) bone area within the central region (i.e., bone through growth) of the AM implants for A and B sections (Figure 3), based on a method previously described. ${ }^{30}$ For each implant, data from A and B sections were combined for analysis. Bone-in-contact assessments are presented as a percentage of available surface length (\%BIC), while bone area assessments are presented as a percentage of the associated available area (\%BA).

\section{Statistical Methods}

Statistical analyses were performed using SAS (version 9.0, Cary, NC) and Excel. SAS PROC MIXED was used to perform linear mixed models using a restricted maximum likelihood estimation method.
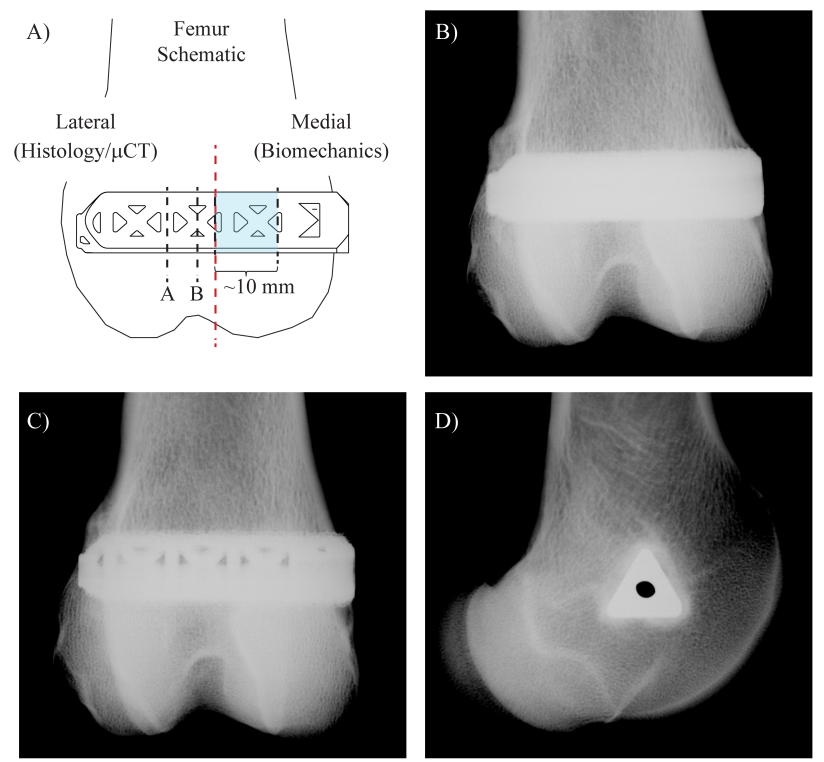

Fig. 2. Schematic of device placement, device geometry, and test plan, highlighting how the implant was sectioned for postmortem evaluations, with the " $A$ " section taken from the most closed section and the " $B$ " section taken from the most open section of the additive manufactured (AM) device (A). The red dashed line indicates where the implant was bisected. Note: the solid titanium plasma spray-coated (TPS) implants were placed and processed in the same respective locations. Representative anteroposterior radiographic images at necropsy of TPS (B) and AM (C) implants. Representative lateral radiographic image at necropsy of both implant types (D).
Models were constructed with time and treatment as fixed effects and animal as a random variable. Analysis was conducted considering data from both timepoints together or stratified by each time-point. The fixed effects estimates were examined for each AM group compared to the TPS-coated implants. When type III tests of fixed effects were significant $(p<0.05)$, a least squares means post hoc comparison was used to look for treatment level effects. Parameters with $p<0.05$ were considered statistically significant. Data are presented as mean \pm standard deviation.

\section{Results}

\section{Implants and Sheep Model}

The AM implants met all design inputs aside from the coating thickness, which due to the printing process was slightly thicker than the design input. All sheep were skeletally mature as confirmed radi-

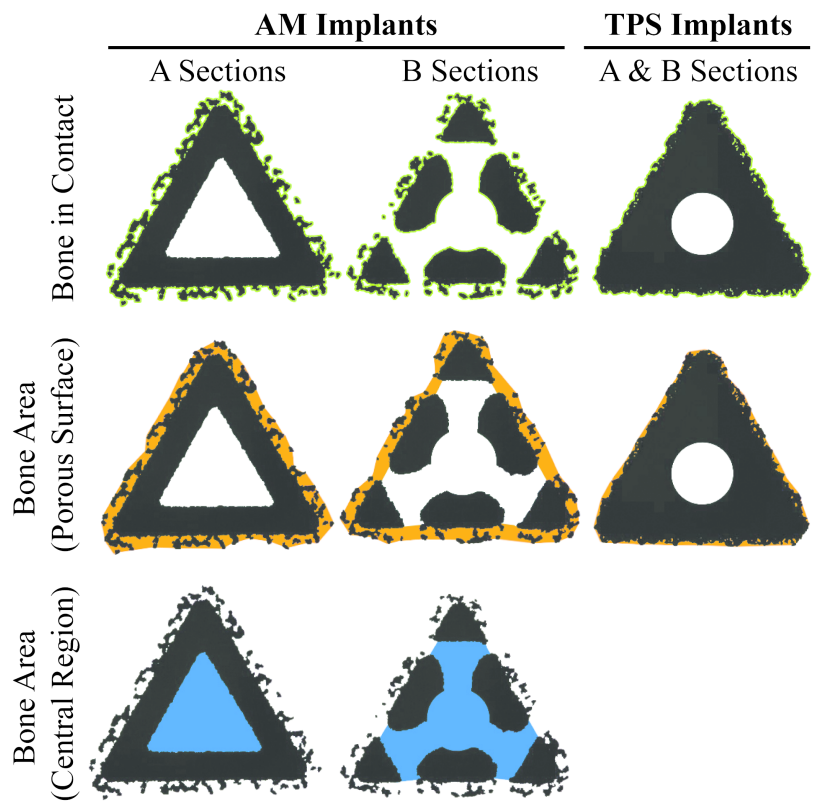

Fig. 3. Schematic of quantitative histomorphometric regions of interest for $A$ and $B$ sections of AM and TPS implants (Figure 2A). Note: $A$ and $B$ sections for TPS implants have the same cross-section. Top row: Green outline indicates the surface length available for bone in contact (BIC) measurements. Pores within the porous coatings were included in the available surface length. Middle row: Orange area indicates the available area for bone to grow into the porous surface and within the open fenestration between the substrate of the B section of the AM implant and the boundary line drawn between the peaks of the porous coating. The area also includes any open pores within the porous coating available for bone to grow in. Bottom row: Blue area indicates the available area for bone to grow into the central region of the AM implants. This metric was not measured for the TPS implants. AM = additive manufactured, TPS = titanium plasma spray. 
ographically. Pre-operative weights were

$93.65 \pm 9.3 \mathrm{~kg}$. No signs of substantial weight change were observed at the time of necropsy, and all sheep completed their in-life phase in good health. All implants were placed without complication. Gross examination at necropsy demonstrated normal postoperative healing. Post-mortem radiographic images showed peri-implant bone formation in all cases (Figure 2B-D).

\section{Biomechanics}

Biomechanical push-out testing showed no significant differences among groups at either time-point for peak push-out force, energy-to-failure, or ultimate shear strength. Peak push-out and energy-tofailure values are presented in Table 1. Ultimate shear strength results are presented in Figure 4A. All metrics were significantly greater at 12 weeks compared to 6 weeks $(p<0.05)$. Gross examination revealed "spot-welds" of bone remaining attached post-pushout testing to TPS implants, whereas a $\sim 2-3 \mathrm{~mm}$ continuous ring of integrated bone remained attached to all AM groups following testing (Figure 4B). Furthermore, tissue was observed growing into the open fenestrations and throughout the core of all AM groups (Figure 4B).

\section{Microcomputed Tomography}

Microcomputed tomography results showed evidence of peri-implant bone around all implants, with dark areas observed around implant corners and central regions (Figure 5). Histologic analysis showed continuous bone formation around the periphery of all implants and within the core of the AM groups (Figure 6), verifying the dark areas observed under $\mu \mathrm{CT}$ to be artifact. Due to this scattering artifact, bone volume and density were not quantified using $\mu \mathrm{CT}$.

\section{Semi-Quantitative Histomorphometry}

Semi-quantitative histomorphometric analysis (Table 2) revealed bone maturation scores at the surface of all treatment groups to be 2 at 6 weeks, i.e. 50:50 lamellar to woven bone. By 12 weeks, the median scores of TPS and AM+Auto implants increased to 3 (50-75\% lamellar bone), while AM and AM+HA groups remained unchanged at 2. At 6 weeks, the median score for bone maturation within the center region of the AM implants was 0 for the AM group,
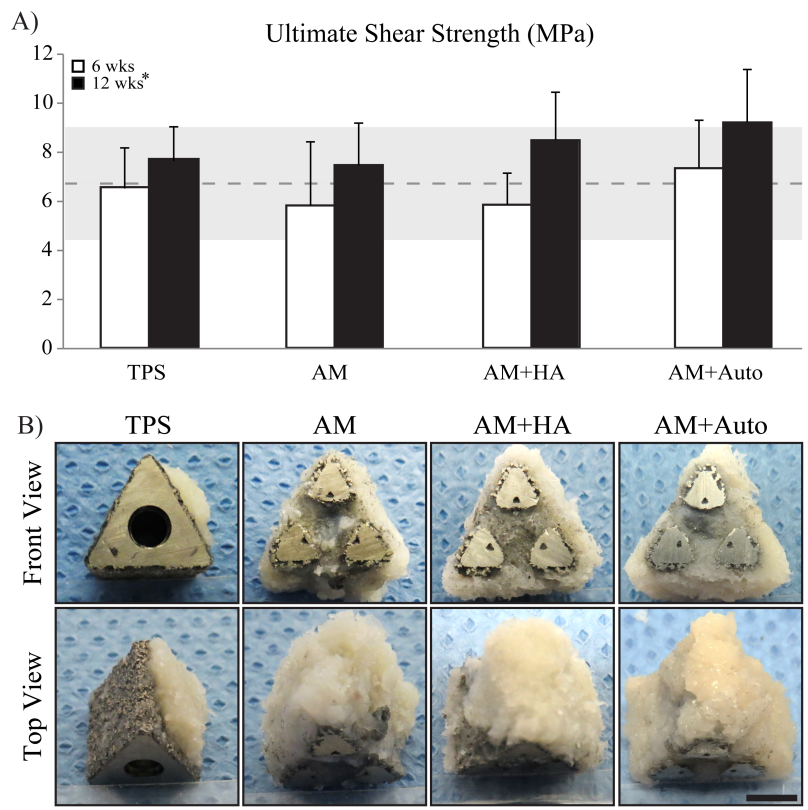

Fig. 4. Biomechanical push-out testing results for ultimate shear strength (A). Dashed line and gray shaded region indicate the average ultimate shear strength and standard deviation, respectively, of bovine cancellous bone. 33 Representative gross images of post-push-out samples at 12 weeks show different failure modes (B). TPS implants failed primarily at the bone-implant interface, with some large "spot-welding" patches remaining, while a $\sim 2-3 \mathrm{~mm}$ ring of bone remained securely attached to all AM groups even after being subjected to $\geq 2500 \mathrm{~N}$. Tissue can also be observed growing into the open fenestrations and through the central core of all AM groups. Gross examination at 6 weeks revealed similar findings. Data are represented as mean \pm standard deviation, $*=p<0.01$ within time-point factor, scale bar $=5 \mathrm{~mm}$. TPS = titanium plasma spray, $A M=$ additive manufactured, $A M+H A=$ additive manufactured with hydroxyapatite, $A M+A u t o=$ additive manufactured with autograft.

Table 1. Push-out Testing Results. Data are presented as Mean \pm Standard Deviation.

\begin{tabular}{|c|c|c|c|c|c|c|c|c|}
\hline & \multicolumn{4}{|r|}{6 weeks } & \multicolumn{4}{|r|}{12 weeks } \\
\hline & TPS & $\mathbf{A M}$ & $\mathbf{A M}+\mathbf{H A}$ & AM+Auto & TPS & $\mathbf{A M}$ & $\mathbf{A M}+\mathbf{H A}$ & AM+Auto \\
\hline Peak Push-Out Force (N) & $2635 \pm 705$ & $2313 \pm 1057$ & $2351 \pm 535$ & $2961 \pm 761$ & $3061 \pm 645$ & $3029 \pm 710$ & $3450 \pm 710$ & $3766 \pm 867$ \\
\hline Energy to Failure (Nmm) & $1497 \pm 537$ & $1209 \pm 623$ & $1144 \pm 513$ & $1660 \pm 710$ & $1699 \pm 507$ & $1603 \pm 549$ & $1971 \pm 748$ & $2188 \pm 543$ \\
\hline
\end{tabular}

TPS = titanium plasma spray, AM = additive manufactured, $A M+H A=$ additive manufactured with hydroxyapatite, $A M+A u t o=$ additive manufactured with autograft. 
while the AM+HA and AM+Auto groups had median scores of 1 (25-50\% lamellar bone). By 12 weeks, median scores increased to 2 for the AM and $\mathrm{AM}+$ Auto groups, while the AM+HA remained unchanged at 1 . Bone fragments from implantation were observed within the porous surfaces of all implants, and within the core of the AM implants,

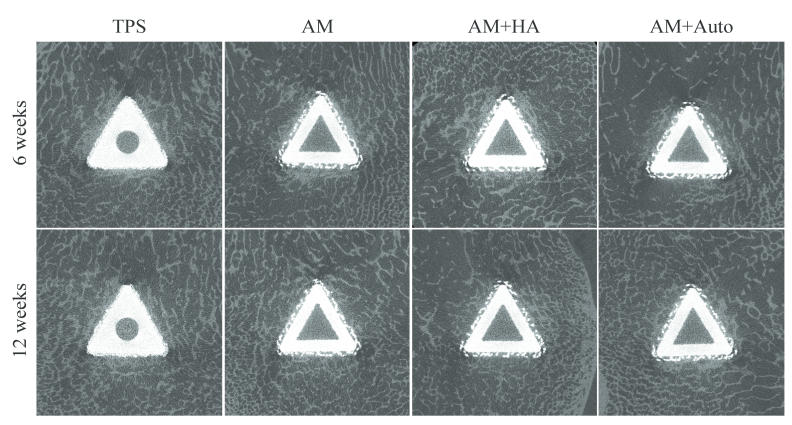

Fig. 5. Microcomputed tomographic images of implants at necropsy, corresponding to the " $\mathrm{A}$ " histological cross-sections. Peri-implant bone is evident around all implants. Scattering artifact can be observed at implant corners. TPS = titanium plasma spray, $A M=$ additive manufactured, $\mathrm{AM}+\mathrm{HA}=$ additive manufactured with hydroxyapatite, $A M+A u t o=$ additive manufactured with autograft.

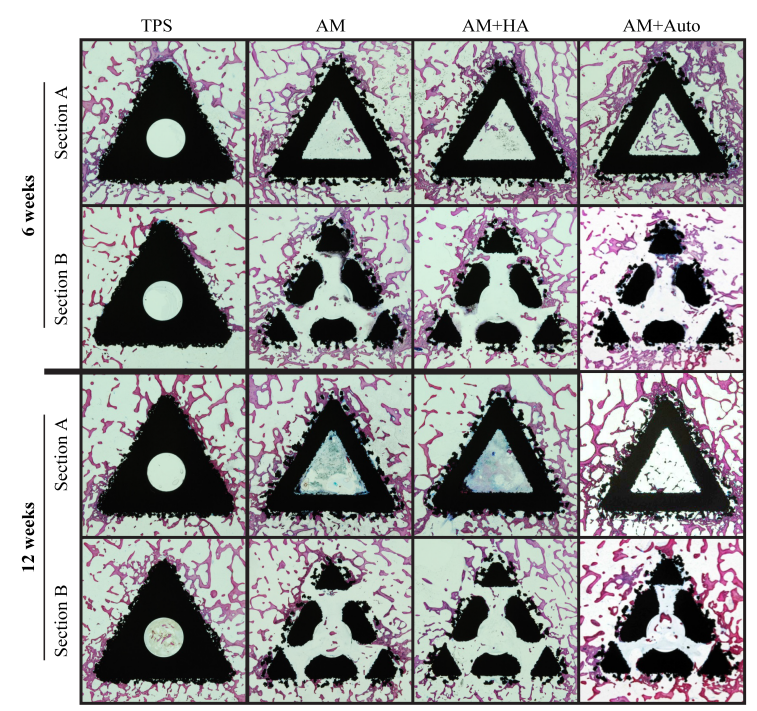

Fig. 6. Representative A and B histological cross-sections from all treatment groups shown at original magnification (1x). Qualitative assessment showed that bone ongrowth/ingrowth to all treatment groups was substantial by 6 weeks and changed with time as the bone matured. Some soft tissue was also seen in contact with the implants, which was mainly composed of a collagen-rich transitional tissue. Bone could also be seen within the central region through the open fenestrations in all AM implant groups. Note that bone forms continuously around the periphery of the implants, and within the open core of the AM implant groups, indicating the dark regions observed under $\mu C T$ to be artifact. TPS = titanium plasma spray, AM = additive manufactured, $\mathrm{AM}+\mathrm{HA}=$ additive manufactured with hydroxyapatite, $A M+A u t o=$ additive manufactured with autograft. demonstrating self-harvesting of host bone. No necrosis and very few inflammatory cells (median scores of 0 ) were seen in any of the sections at either time point, providing no evidence of a safety issue.

\section{Quantitative Histomorphometry}

Quantitative histomorphometric analysis results are presented in Table 3. At 6 weeks, \%BIC was found to be significantly greater in TPS implants compared to all other groups $(p<0.003)$, with no other differences among groups. By 12 weeks, there were no significant differences in \%BIC among groups. Time was a significant factor toward increasing $\% \mathrm{BIC}$ at 12 weeks $(p<0.004)$. At 6 weeks, $\% \mathrm{BA}$ within the porous surfaces was found to be significantly greater in TPS implants compared to all other groups $(p<0.01)$. By 12 weeks, there were no significant differences in \%BA within the porous surfaces among TPS, AM and $\mathrm{AM}+$ Auto groups, with $\mathrm{AM}+\mathrm{HA}$ implants having significantly less \%BA within their porous surfaces compared to TPS implants at this time $(p<0.03)$. At 6 weeks, \% BA within the center region was found be significantly greater in AM+Auto implants compared to AM $(p<0.049)$ and AM+HA $(p<0.04)$ implants.

By 12 weeks, the AM+HA implants $(p<0.01)$ had significantly lower \%BA within the center compared to $\mathrm{AM}+$ Auto implants. Time was a significant factor $(p<0.01)$.

By design, the porous surface of the AM implants offered comparatively greater available surface length and area. To quantify these differences, the histologic A sections of the TPS and AM implants were compared, revealing that the AM surface provides $1.4 \mathrm{x}$ the available surface length $(\mathrm{p}<0.0001)$ and $2.8 \mathrm{x}$ the available area $(\mathrm{p}<0.0001)$ for osteointegration compared to the TPS surface per Student's $t$-test (Figure 7A-B). These significant differences in surface structure merited additional analysis on data not normalized to surface length or surface area to compare the amount of bone contacting the surface and growing within the porous surface/central region for the different implant groups (Table 3). Analyzing the data as such showed no difference in BIC among groups at either time point, with time again being a significant factor $(p<0.001)$. On the other hand, at 6 weeks, BA filling the porous surfaces was found to be significantly greater in all AM groups compared to 
TPS implants $(p<0.02)$. By 12 weeks, the AM+Auto group had significantly more BA within the porous surface compared to the TPS implants $(p<0.001)$ and $\mathrm{AM}+\mathrm{HA}$ implants $(p<0.01)$, with the AM and $\mathrm{AM}+\mathrm{HA}$ groups also having significantly greater $\mathrm{BA}$ within the porous surface than TPS implants at this time $(p<0.04)$. Finally, trends for BA within the center region were similar to those calculated for $\% \mathrm{BA}$ in this region.

To analyze the contribution of the open fenestrated core of the AM groups, BA in the porous surface plus the central region of the AM groups were also calculated and compared to the BA of TPS implants (Figure 7C). At 6 weeks, total BA was found to be significantly greater in the AM+Auto implants compared to TPS $(p<0.0001), \mathrm{AM}(p<0.02)$, and $\mathrm{AM}+\mathrm{HA}(p<0.02)$ groups. The AM and AM+HA groups also had significantly more total BA than TPS $(p<0.005)$ implants at this time. By 12 weeks, AM and AM+Auto implants had significantly greater total BA than $\mathrm{AM}+\mathrm{HA}$ implants $(p<0.03)$, which in turn had significantly more total BA than TPS implants $(p<0.01)$. Overall, the AM, AM+HA, and $\mathrm{AM}+$ Auto groups had 2.5x, 2.0x, and 3.3x more bone filling the implants, respectively, compared to the

Table 2. Semi-Quantitative Histological Analysis Results. Data are presented as Median [Range].

\begin{tabular}{|l|r|r|r|r|r|r|r|r|}
\hline & \multicolumn{9}{|r|}{$\mathbf{6}$ weeks } & \multicolumn{3}{|c|}{ weeks } \\
\cline { 2 - 8 } & TPS & AM & AM+HA & AM+Auto & TPS & AM & AM+HA & AM+Auto \\
\hline Bone Maturation at Surface & $2[0-4]$ & $2[1-3]$ & $2[1-2]$ & $2[2-2]$ & $3[1-4]$ & $2[1-3]$ & $2[1-4]$ & $3[2-4]$ \\
\hline Bone Maturation within Center & N/A & $0[0-2]$ & $1[0-2]$ & $1[0-3]$ & N/A & $2[0-3]$ & $1[0-2]$ & $2[0-4]$ \\
\hline
\end{tabular}

TPS = titanium plasma spray, $\mathrm{AM}=$ additive manufactured, $\mathrm{AM}+\mathrm{HA}=$ additive manufactured with hydroxyapatite, $\mathrm{AM}+\mathrm{Auto}=$ additive manufactured with autograft. Scale is as follows: $0=<25 \%$ lamellar bone, $1=25-50 \%$ lamellar bone, $2=50: 50$ lamellar to woven bone, $3=50-75 \%$ lamellar bone, $4=>75 \%$ lamellar bone.

Table 3. Quantitative Histomorphometric Analysis Results. Data are presented as Mean (Standard Deviation).

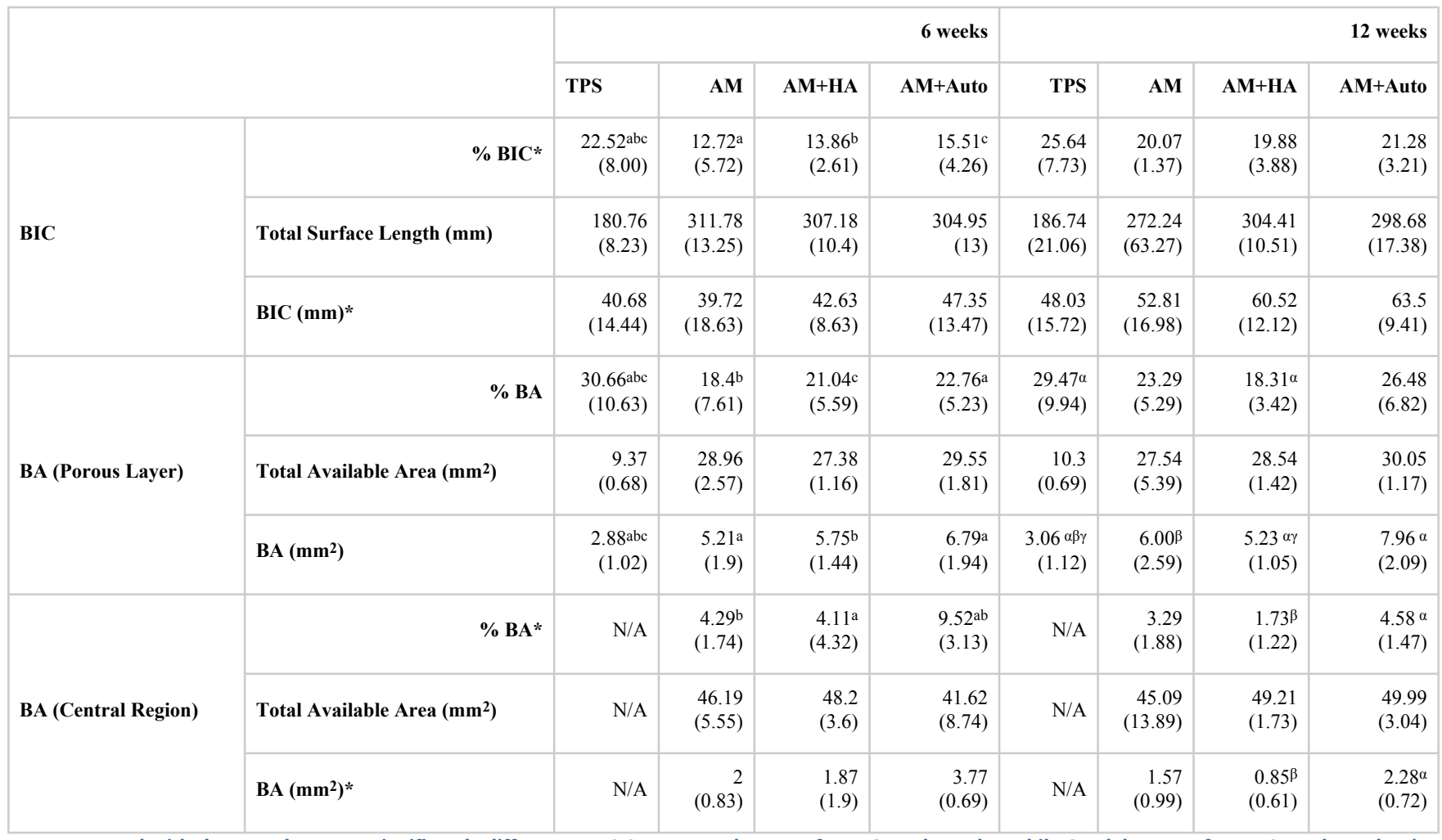

Data connected with the same letter are significantly different $(p<0.05)$. Roman letters refer to 6 week results, while Greek letters refer to 12 week results. * $=$ $p<0.05$ within time-point factor. TPS = titanium plasma spray, $A M=$ additive manufactured, $A M+H A=$ additive manufactured with hydroxyapatite, $A M+A u t o=$ additive manufactured with autograft, $\mathrm{BIC}=$ bone-in-contact, $\mathrm{BA}=$ bone area.

Downloaded from http://ijssurgery.com/ by guest on April 26, 2023 
TPS implants at 12 weeks.
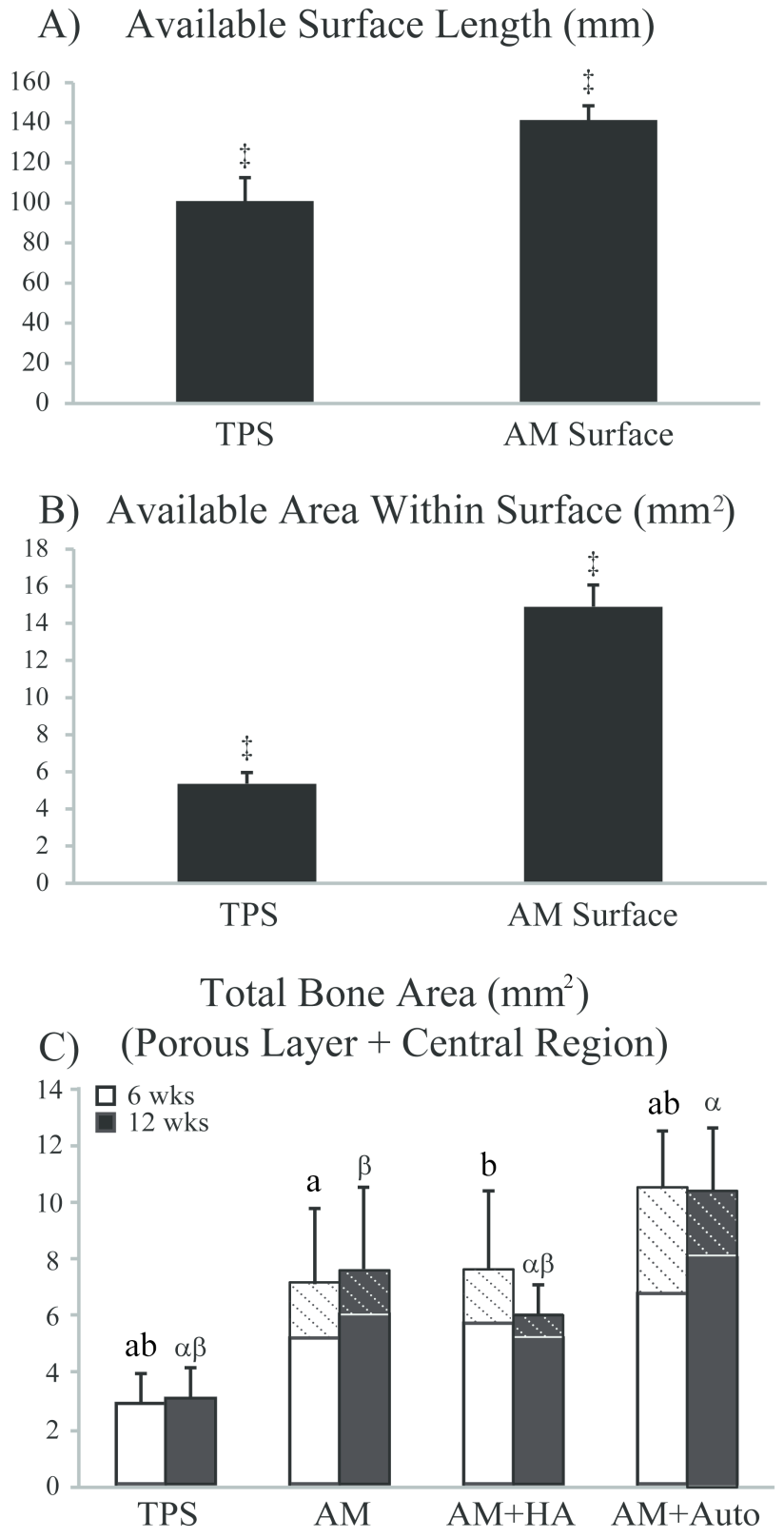

Fig. 7. Quantitative histomorphometric results of the outer surface of $A$ sections for available surface length $(A)$ and available area within the surface (B). Results for both metrics were significantly greater in the AM surfaces (averaged across all three AM implant groups) compared to the TPS implants $(¥=p<0.0001)$. Bone area (BA) within the porous surface and central region is shown in (C). For AM implant groups, the "hashed" regions of (C) signify the contribution of BA within the central region, whereas the "unhashed" regions signify the contribution of BA within the porous surface. Bars having the same letter are significantly different $(p<$ 0.05). Roman letters refer to 6 week results, while Greek letters refer to 12 week results. Data are represented as mean \pm standard deviation. TPS = titanium plasma spray, $A M=$ additive manufactured, $A M+H A=$ additive manufactured with hydroxyapatite, $A M+$ Auto $=$ additive manufactured with autograft.

\section{Discussion}

This study compared the in vivo bone response to traditional TPS-coated implants versus fenestrated AM implants. As hypothesized, AM implants with or without HA coating or autograft displayed similar biomechanical and, in some cases, enhanced histomorphometric results compared to the TPS-implant, similar to results achieved in vitro (see Part I). Further, gross examination and microscopic evaluation showed bone through growth into the core of the AM implants. The nanocrystalline HA coating was not found to further promote osteointegration, corroborating results previously determined in vitro (see Part I). Placing autograft onto and into the AM implants, however, was found to be advantageous, as anticipated. Specifically, AM+Auto implants had the greatest (although not significant) push-out measurements and significantly greater bone ongrowth/ingrowth compared to all other groups, suggesting the $\mathrm{AM}+$ Auto group may further promote osteointegration. While all implants investigated formed strong, stable interfaces with host bone, the AM+Auto group data suggests that addition of autograft to AM implants may produce an even more fortified boneimplant interface.

In the present study, quantitative histomorphometric analysis normalized to available surface length or surface area found the TPS-implants to have significantly greater $\% \mathrm{BIC}$ and $\% \mathrm{BA}$ within the porous surface than all other groups at 6 weeks (Table 3 ). These results, however, were not consistent with push-out data, as the significantly greater \% BIC for the TPS implants did not result in significantly different biomechanics results at this time point. Although normalizing BIC and BA to available surface length and area, respectively, is the most common means to analyze quantitative histomorphometric data, the significant differences in the available surface length and area between the TPS and AM surfaces may suggest BIC and $\mathrm{BA}$ are more appropriate comparative metrics for this study. Viewing the data in this way showed no difference in BIC among groups, consistent with the biomechanics results at either time point. Notably, at 12 weeks, the ultimate shear strength of the $\mathrm{AM}+$ Auto group was $1.2 \mathrm{x}$ greater than TPS implants $(p=0.050)$, whereas the BIC of the AM+Auto group 
was $1.3 x$ greater than TPS implants $(p=0.050)$ at this time point. BA within the porous surface and total $\mathrm{BA}$, on the other hand, were found to be significantly greater in all AM groups compared to the TPS implants, with $\mathrm{AM}+$ Auto implants having the greatest values at both time points (Figure $7 \mathrm{C}$ ). Thus, the AM implants all experienced significantly more bony ongrowth, ingrowth, and through growth compared to the TPS implants, which correlates with the differing biomechanical failure modes between implant designs (Figure 5C). A study on implant stability in rabbit tibia showed comparable results, finding similar trends between implant stability assessed via resonance frequency and bone-implant contact area for titanium screws. ${ }^{31}$ While both the TPS and AM implants formed stable interfaces with host bone, these results indicate that they each have distinct mechanisms for promoting implant stabilization that may influence the degree of biological fixation.

The differences in porous surface structure and the solid versus fenestrated architecture of the TPS and AM implants may further explain the distinctive push-out failure mechanisms. Specifically, while the TPS implants failed primarily at the bone-implant interface, a $\sim 2-3 \mathrm{~mm}$ ring of continuous and integrated host bone remained securely attached to all three AM implant groups (Figure 5C). The decreased depth of bone ingrowth into the TPS coating likely resulted in the bone failing more cleanly from the surface. The deeper bone ingrowth into highly interconnected pores apparent throughout the full thickness of the AM surface, as well as the additional bone through growth into the fenestrations, likely resulted in an enhanced bone interlock throughout the AM implant's entire structure. These results are consistent with a study that found the ultimate shear strength of AM titanium rods with a porous surface to be significantly greater than TPS-coated rods in ovine tibial cortical bone at both 4 and 12 weeks. ${ }^{32}$ Bertollo et al. attributed their results to greater osteointegration into the more porous surface of their AM scaffolds. ${ }^{32}$ Although the present study did not similarly find statistical differences in the push-out testing of the AM versus TPS implants, this may be due to fact that the testing was conducted on segments fully surrounded by cancellous bone. While the ultimate shear strength of sheep cancellous bone has yet to be reported, the ultimate shear strength of bovine femoral cancellous bone has been measured at $6.57 \pm 2.46 \mathrm{MPa} .{ }^{33}$ Looking to Figure $5 \mathrm{~A}$, it can be seen that the majority of the tested sample's ultimate shear strengths were within the range of cancellous bone. Unlike the TPS implants, the bone-implant interface of all the AM groups remained intact after $\geq$ $2500 \mathrm{~N}$ of force; this indicates that the bone-implant interface is stronger that than the surrounding cancellous bone. Taken together, the differing push-out failure mechanisms encountered in the present study may demonstrate that the integrity of the bone integration of AM implants with cancellous bone is greater than that of the TPS implants.

It is known that the local mechanical environment in the healing peri-implant interface tissue stimulates cellular differentiation and tissue synthesis. ${ }^{34} \mathrm{~A}$ study in rabbit femoral condyles found host bone integrated more rapidly with sintered porous-surfaced implants during early healing compared to TPS-coated implants. ${ }^{35} \mathrm{~A}$ follow-up finite element analysis found the comparatively more open porous structure of the sintered porous-surfaces provided a local mechanical environment that favored mineralization compared to the less porous surface of the TPS-coating. ${ }^{36} \mathrm{Giv}$ en the more open and interconnected nature of the AM porous surfaces, it is possible that the tissue level stresses and strains are more favorable around the fenestrated AM implants compared to the TPS implants. Viewing bone formation around the different implant designs in Figure 8 corroborates this theory. While bone surrounding the TPS-coated implants is less-organized and more compacted than native trabecular bone, bone surrounding the AM implants is organized in a more normal trabecular formation, with bone struts aligning with the porous surface features. This could indicate a more physiological loadtransfer through the AM porous surface compared to the TPS-coated surface, although, given the differences in load transfer in this model compared to that in humans, the clinical implications of this finding warrant further investigation. However, a more open, fenestrated design of the AM implants compared to the solid-based TPS implants has the potential for a more physiological interface with cancellous bone that, based on the previous rabbit study, ${ }^{35,36}$ potentially integrates more rapidly and effectively. 
There are certain limitations to the present in vivo study that should be considered. An ovine model is widely used to investigate orthopedic implants, as it displays similar bone ingrowth and mineral apposition rates to humans, ${ }^{37}$ and its size accommodates clinically-sized implants, ${ }^{38}$ although sheep do have comparatively greater bone density. ${ }^{39}$ While the present results are from a healthy bone environment, the increased bone integration and better load transmission of the AM implants may prove beneficial compared to solid TPS implants in instances of impaired bone health ${ }^{40}$ meriting future work in this area. Further, the implants in this model were placed in the
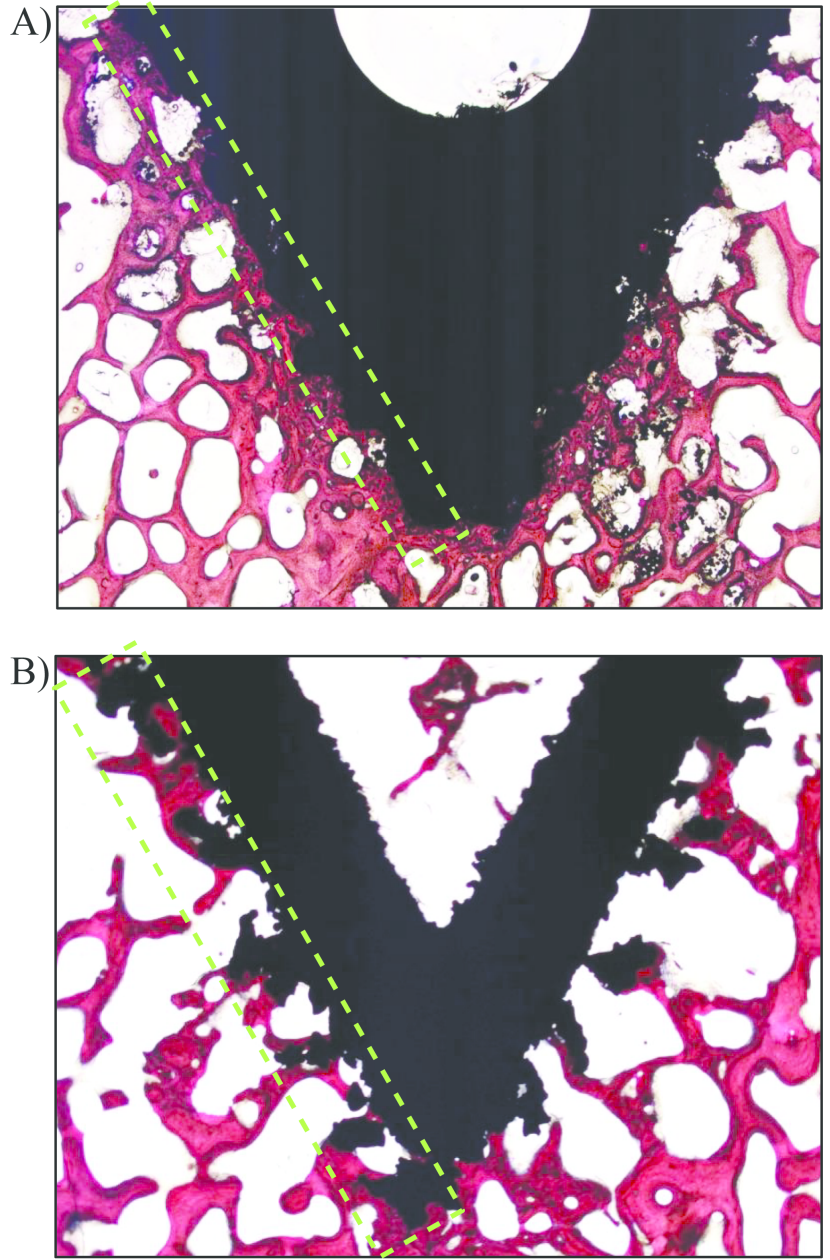

Fig. 8. Representative images of titanium plasma spray-coated (TPS) (A) and additive manufactured (AM) (B) implant surfaces (magnification of $7 \mathrm{x}$ ), with green-dashed region highlighting the differences in porous surface features between the two implant designs. Note that the porous surface of the AM implants is thicker than that of the TPS-coating. New bone can be seen within the open portion of the AM implant. The trabecular morphology around the AM implant is well-organized and aligns with the porous features of the surface, whereas a more compacted morphology is evident around the TPS-coated implant. This may indicate more physiological load-transfer through the AM implant compared to the TPS-coated implant. distal femur; however, the clinical utility of these implants is to promote SI joint stabilization and fusion. Although it remains unknown how the AM implants would respond if placed across the SI joint, it is hypothesized that the additional loads and loading schemes would require greater implant-bone stability. The combined bony integration onto, into, and through the AM implant may therefore be more stable and reliable than surface growth alone in the TPS implants across the joint environment. Future studies should be conducted to test this hypothesis.

Overall, results presented in this study corroborate previous clinical investigations of the TPS implant. ${ }^{8-10,41,42}$ Specifically, the TPS implants were found to exhibit a substantial degree of bone ongrowth and ingrowth to their surfaces, resulting in a mechanically stable interface with surrounding host bone. The AM implants investigated herein provide a highly interconnected porous surface with an open fenestrated core structure that offers comparatively greater surface area for bony integration and the ability to be pre-packed with graft material, which may benefit a number of spinal implants, such as fusion, disc, and SI joint implants. These AM implants likewise demonstrated substantial bone ongrowth and ingrowth to their porous surfaces, with additional bone through growth in the cores. Although similar HA coatings have previously demonstrated a beneficial effect on non-porous titanium surfaces or inert structures, such as PEEK, ${ }^{26,27}$ the current study showed that the dip, spin, heat treated nanocrystalline HA coating did not further enhance results compared to uncoated AM implants. The addition of autograft, on the other hand, resulted in significantly increased bone ingrowth and through growth within the AM implant, potentially further promoting osteointegration. As a whole, the AM implants experienced significantly more bony infiltration compared to the TPS implants. Taken together, the AM implant has the potential of promoting an enhanced boneimplant interface compared to TPS-coated implants that could provide additional stabilization upon implantation.

\section{Acknowledgements}

Special thanks to Bret Schneider (employee of SI- 
BONE, Inc.) who designed and developed the additive manufactured implants investigated in this study and to Daniel Cher, MD (employee of SI-BONE, Inc.) for his help in developing and conducting the statistical analyses for this study.

\section{References}

1. Shmagel A, Foley R, Ibrahim H. Epidemiology of chronic low back pain in US adults: National Health and Nutrition Examination Survey 2009-2010.

Arthritis Care Res. 2016 Mar 18.

2. United States Bone and Joint Initiative: The Burden of Musculoskeletal Diseases in the United States (BMUS), [Internet]. Third Edition. 2014 [cited 2016 Aug 19]. Available from: Available at http://www.boneandjointburden.org.

3. Schwarzer AC, Aprill CN, Bogduk N. The sacroiliac joint in chronic low back pain. Spine. 1995 Jan 1;20(1):31-7.

4. Sembrano JN, Polly DW. How often is low back pain not coming from the back? Spine. 2009 Jan 1;34(1):E27-32.

5. Bernard TN, Kirkaldy-Willis WH. Recognizing specific characteristics of nonspecific low back pain. Clin Orthop. 1987 Apr;(217):266-80.

6. Maigne JY, Aivaliklis A, Pfefer F. Results of sacroiliac joint double block and value of sacroiliac pain provocation tests in 54 patients with low back pain. Spine. 1996 Aug 15;21(16):1889-92.

7. Irwin RW, Watson T, Minick RP, Ambrosius WT. Age, Body Mass Index, and Gender Differences in Sacroiliac Joint Pathology. Am J Phys Med Rehabil. 2007 Jan;86(1):37-44.

8. Polly DW, Swofford J, Whang PG, Frank C, Glaser JC, Limoni RP, et al. Two-Year Outcomes from a Randomized Controlled Trial of Minimally Invasive Sacroiliac Joint Fusion vs. Non-Surgical Management for Sacroiliac Joint Dysfunction. Int J Spine Surg. 2016 Aug 23;10:Article 28.

9. Sturesson B, Kools D, Pflugmacher R, Gasbarrini A, Prestamburgo D, Dengler J. Six-month outcomes from a randomized controlled trial of minimally invasive SI joint fusion with triangular titanium implants vs conservative management. Eur Spine J Off Publ Eur Spine Soc Eur Spinal Deform Soc Eur Sect Cerv Spine Res Soc. 2016 May 14;
10. Duhon BS, Bitan F, Lockstadt H, Kovalsky D, Cher D, Hillen T, et al. Triangular Titanium Implants for Minimally Invasive Sacroiliac Joint Fusion: 2-Year Follow-Up from a Prospective Multicenter Trial. Int J Spine Surg. 2016;10:13.

11. Cher DJ, Reckling WC, Capobianco RA. Implant survivorship analysis after minimally invasive sacroiliac joint fusion using the iFuse Implant System. Med Devices Evid Res. 2015 Nov 23;8:485-92. 12. Martin BI, Mirza SK, Comstock BA, Gray DT, Kreuter W, Deyo RA. Reoperation rates following lumbar spine surgery and the influence of spinal fusion procedures. Spine. 2007 Feb 1;32(3):382-7. 13. Spector M. Historical review of porous-coated implants. J Arthroplasty. 1987;2(2):163-77.

14. Hahn H, Palich W. Preliminary evaluation of porous metal surfaced titanium for orthopedic implants. J Biomed Mater Res. 1970 Dec;4(4):571-7. 15. Cook SD, Barrack RL, Thomas KA, Haddad RJ Jr. Quantitative analysis of tissue growth into human porous total hip components. J Arthroplasty. 1988;3(3):249-62.

16. Traynelis VC. Cervical arthroplasty. Clin Neurosurg. 2006;53:203-7.

17. Yoon BJV, Xavier F, Walker BR, Grinberg S, Cammisa FP, Abjornson C. Optimizing surface characteristics for cell adhesion and proliferation on titanium plasma spray coatings on PEEK. Spine J [Internet]. 2016 May [cited 2016 Jun 3]; Available from: http://linkinghub.elsevier.com/retrieve/pii/ S1529943016302030

18. Yang YZ, Tian JM, Tian JT, Chen ZQ, Deng $\mathrm{XJ}$, Zhang DH. Preparation of graded porous titanium coatings on titanium implant materials by plasma spraying. J Biomed Mater Res. 2000

Nov;52(2):333-7.

19. Asaoka K, Kuwayama N, Okuno O, Miura I. Mechanical properties and biomechanical compatibility of porous titanium for dental implants. J Biomed Mater Res. 1985 Aug;19(6):699-713.

20. Ryan G, Pandit A, Apatsidis DP. Fabrication methods of porous metals for use in orthopaedic applications. Biomaterials. 2006 May;27(13):2651-70. 21. Hutmacher DW. Scaffolds in tissue engineering bone and cartilage. Biomaterials. 2000

Dec;21(24):2529-43.

22. Karageorgiou V, Kaplan D. Porosity of 3D bio- 
material scaffolds and osteogenesis. Biomaterials. 2005 Sep;26(27):5474-91.

23. Wang X, Xu S, Zhou S, Xu W, Leary M, Choong $\mathrm{P}$, et al. Topological design and additive manufacturing of porous metals for bone scaffolds and orthopaedic implants: A review. Biomaterials. 2016 Mar;83:127-41.

24. Sun L, Berndt CC, Gross KA, Kucuk A. Material fundamentals and clinical performance of plasmasprayed hydroxyapatite coatings: a review. J Biomed Mater Res. 2001;58(5):570-92.

25. Sun L, Berndt CC, Gross KA, Kucuk A. Material fundamentals and clinical performance of plasmasprayed hydroxyapatite coatings: a review. J Biomed Mater Res. 2001;58(5):570-92.

26. Barkarmo S, Wennerberg A, Hoffman $M$, Kjellin $\mathrm{P}$, Breding K, Handa $\mathrm{P}$, et al. Nanohydroxyapatite-coated PEEK implants: a pilot study in rabbit bone. J Biomed Mater Res A. 2013 Feb;101(2):465-71.

27. Meirelles L, Arvidsson A, Andersson M, Kjellin P, Albrektsson T, Wennerberg A. Nano hydroxyapatite structures influence early bone formation. J Biomed Mater Res A. 2008 Nov;87(2):299-307.

28. Heinl P, Müller L, Körner C, Singer RF, Müller FA. Cellular Ti-6Al-4V structures with interconnected macro porosity for bone implants fabricated by selective electron beam melting. Acta Biomater. 2008 Sep;4(5):1536-44.

29. Lopes MA, Santos JD, Monteiro FJ, Ohtsuki C, Osaka A, Kaneko S, et al. Push-out testing and histological evaluation of glass reinforced hydroxyapatite composites implanted in the tibia of rabbits. J Biomed Mater Res. 2001 Mar 15;54(4):463-9.

30. Sumner DR, Jasty M, Jacobs JJ, Urban RM, Bragdon CR, Harris WH, et al. Histology of porouscoated acetabular components. 25 cementless cups retrieved after arthroplasty. Acta Orthop Scand. 1993 Dec;64(6):619-26.

31. Meredith N, Shagaldi F, Alleyne D, Sennerby L, Cawley P. The application of resonance frequency measurements to study the stability of titanium implants during healing in the rabbit tibia. Clin Oral Implants Res. 1997 Jun;8(3):234-43.

32. Bertollo N, Da Assuncao R, Hancock NJ, Lau A, Walsh WR. Influence of Electron Beam Melting Manufactured Implants on Ingrowth and Shear
Strength in an Ovine Model. J Arthroplasty. 2012 Sep;27(8):1429-36.

33. Garrison JG, Gargac JA, Niebur GL. Shear strength and toughness of trabecular bone are more sensitive to density than damage. J Biomech. 2011 Nov 10;44(16):2747-54.

34. Pauwels F. Biomechanics of the Locomotor Apparatus Contributions on the Functional Anatomy of the Locomotor Apparatus [Internet]. Berlin, Heidelberg: Springer Berlin Heidelberg; 1980 [cited 2016 May 3]. Available from: http://dx.doi.org/10.1007/ 978-3-642-67138-8

35. Simmons CA, Valiquette N, Pilliar RM. Osseointegration of sintered porous-surfaced and plasma spray-coated implants: An animal model study of early postimplantation healing response and mechanical stability. J Biomed Mater Res. 1999

Nov;47(2):127-38.

36. Simmons CA, Meguid SA, Pilliar RM. Differences in osseointegration rate due to implant surface geometry can be explained by local tissue strains. J Orthop Res Off Publ Orthop Res Soc. 2001 Mar;19(2):187-94.

37. Willie BM, Bloebaum RD, Bireley WR, Bachus $\mathrm{KN}$, Hofmann AA. Determining relevance of a weight-bearing ovine model for bone ingrowth assessment. J Biomed Mater Res A. 2004 Jun 1;69(3):567-76.

38. Martini L, Fini M, Giavaresi G, Giardino R. Sheep model in orthopedic research: a literature review. Comp Med. 2001 Aug;51(4):292-9.

39. Aerssens J, Boonen S, Lowet G, Dequeker J. Interspecies differences in bone composition, density, and quality: potential implications for in vivo bone research. Endocrinology. 1998 Feb;139(2):663-70. 40. Marco F, Milena F, Gianluca G, Vittoria O. Peri-implant osteogenesis in health and osteoporosis. Micron. 2005 Oct;36(7-8):630-44.

41. Rudolf L, Capobianco R. Five-year clinical and radiographic outcomes after minimally invasive sacroiliac joint fusion using triangular implants. Open Orthop J. 2014;8:375-83.

42. Polly DW, Cher DJ, Wine KD, Whang PG, Frank CJ, Harvey CF, et al. Randomized Controlled Trial of Minimally Invasive Sacroiliac Joint Fusion Using Triangular Titanium Implants vs Nonsurgical Management for Sacroiliac Joint Dysfunction: 
12-Month Outcomes. Neurosurgery. 2015

Nov;77(5):674-90; discussion 690-1.

\section{Disclosures \& COI}

This study was funded by SI-BONE, Inc. RFM, DPL, and SAY are employees of SI-BONE, Inc. MIG is a consultant of SI-BONE, Inc. There are no other conflicts to report.

\section{Corresponding Author}

Regina F. MacBarb, Ph.D., SI-BONE, Inc., 3055 Olin Ave, Suite 2200, San Jose, CA 95128, USA, gmacbarb@si-bone.com.

Published 1 June 2017.

This manuscript is generously published free of charge by ISASS, the International Society for the Advancement of Spine Surgery. Copyright @ 2017 ISASS. To see more or order reprints or permissions, see http://ijssurgery.com. 